\title{
Prevention and treatment of cystitis during menopause: efficacy of a nutraceutical containing D-mannose, inulin, cranberry, bearberry, Olea europaea, Orthosiphon and Lactobacillus acidophilus
}

\author{
Giampaolo Mainini ${ }^{1}$, Mario Passaro $^{2}$, Antonio Schiattarella ${ }^{3}$, Pasquale De Franciscis ${ }^{3}$, \\ Mariano C. Di Donna ${ }^{4}$, Gennaro Trezza ${ }^{5}$ \\ ${ }^{1}$ Menopausal Center, San Leonardo Hospital, ASL NA3 SUD, Castellammare di Stabia, Naples, Italy \\ ${ }^{2}$ ASL NA3 SUD, Naples, Italy \\ ${ }^{3}$ Unit of Gynecology and Obstetrics, Department of Woman, Child and General and Specialized Surgery, University of Campania \\ "Luigi Vanvitelli", Naples, Italy \\ ${ }^{4}$ Department of Gynecologic Oncology, Arnas Civico, University of Palermo, Palermo, Italy \\ ${ }^{5}$ Unit of Gynecology and Obstetrics, Rummo Hospital, Benevento, Italy
}

\section{Abstract}

Aim of the study: To evaluate the efficacy of a nutraceutical compound containing Uticlin ${ }^{\circledR}$ (D-mannose, cranberry, bearberry, Olea europaea), inulin, Orthosiphon and Lactobacillus acidophilus, in preventing recurrent urinary tract infections (UTIs) in menopausal women.

Material and methods: This was a prospective cohort study of menopausal women recruited with a history of recurrent UTIs in the previous twelve months and who intended to treat their bladder problem without the use of antibiotics and/or anti-inflammatories. Women were proposed the use of an oral nutraceutical compound. The drug was taken for ten days, every month. Women were assigned to two parallel cohorts: patients using (group 1) or not using (group 2) this nutraceutical compound. The primary objective of the study was to evaluate the number of women with less than two infective episodes in the 6-month follow-up and less than three episodes in the 12-month follow-up. The secondary endpoints were to evaluate the reduction of related symptoms at 12-month follow-up, according to the Visual Analog Scale (VAS).

Results: At 6 months of therapy, the reduction in the number of patients with $\geq 2$ UTIs was statistically significant $(p<0.05)$ compared to baseline only in group 1 . At 12 months, the number of patients who were disease-free was significantly higher $(p<0.05)$ in group 1 compared to group 2 . Moreover, the improvement of related symptoms was statistically significant $(p<0.05)$ in group 1 , as shown by the reduction in the VAS scale value.

Conclusions: In menopausal women, the combination of D-mannose, inulin, cranberry, bearberry, Olea europaea, Orthosiphon and Lactobacillus acidophilus SGL 11 per os represents a useful therapy for recurrent UTIS in women wanting a natural approach.

Key words: menopause, cystitis, urinary tract infections, UTIs, D-mannose, nutraceutical.

\section{Introduction}

Urinary tract infections (UTIs) represent a common problem during menopause. About $35-40 \%$ of women experience at least one UTI episode requiring antibiotic treatment and the most common bacteria is Escherichia coli, in $85 \%$ of cases $[1,2]$. The fall of estrogen levels, during menopause, and its impact on urogenital mucosa predispose to bladder infections [3, 4]. Recurrent UTIs severely impact daily life in several activities such as social, sexual and working tasks. The genitourinary syndrome of menopause is characterized by various meno- pausal symptoms, including not only genital and sexual symptoms but also urinary complaints such as urgency, dysuria, and recurrent UTIs [3, 5, 6]. About one-third of these women could develop recurrent infections, that is two or more episodes within six months or three or more episodes within 12 months documented by significant positive urine cultures ( $>10^{3} \mathrm{CFU} / \mathrm{ml}$ ) [7]. E. coli infection increases the risk of developing a recurrent episode [8]. In order to reduce bacterial resistance, it is fundamental to optimize the antibiotic strategy. Drinking at least 1.5 liters of water per day could be a valid 
therapy, but often it is not enough. It is therefore recommended to follow the standard rules of prevention: control constipation; carry out antibiotic therapy only if necessary; use supplements that naturally acidify the urine and/or remove the pathogens from the bladder mucosa [9]. However, women are starting to seek a more natural approach to UTI treatment, and several compounds have been created to respond to this aim. The control of constipation is obtained thanks to prebiotics such as inulin and Lactobacillus acidophilus. Inulin is a polysaccharide produced by many types of plants with digestive and intestinal regulating properties [10, 11]. Lactobacillus acidophilus is a Gram-positive bacterium, able to produce lactic acid that inhibits the growth of pathogenic microorganisms [12, 13]. D-mannose is a monosaccharide, and it seems able to reduce the bacteria adhesion to the urothelium, especially $E$. coli that has filamentous appendices. Some of these appendices are mannose-sensitive, and so the presence of mannose in the bladder could detach them from the bladder wall [14-16]. Cranberry can acidify urine and so inhibits the adhesion of microorganisms to the cell membranes of the urinary epithelium of the host, due to the high content in proanthocyanidins, and favor their elimination through urine [17-19]. Bearberry is a natural antimicrobic for the urinary tract. Once filtered and excreted with urine, it exerts an antiseptic action on the bladder and urethra [20]. Olea europaea is the common olive tree. It is rich in oleuropein, a bitter glucoside especially present in their leaves. For this reason, olive leaves have depurative, anti-inflammatory and with laxative properties $[21,22]$. Orthosiphon, also known as Java tea, thanks to its diuretic action, it is useful in UTIs of bacterial and inflammatory character $[23,24]$. The aim of this study was to assess whether the oral therapy with a nutraceutical containing D-mannose, inulin, cranberry, bearberry, Olea europaea, Orthosiphon and Lactobacillus acidophilus SGL 11 could be useful in the prevention of recurrent UTIs in menopausal women.

\section{Material and methods}

This was a prospective cohort study of women recruited at the Menopause Center of San Leonardo Hospital in Naples, Italy, from January 2018 to January 2019, with history of recurrent UTIs (three or more episodes within 12 months documented by urine cultures $>10^{3} \mathrm{CFU} / \mathrm{ml}$ ) and who intended to treat their bladder problem without the use of antibiotics and/or anti-inflammatories. Exclusion criteria were: pelvic organ prolapse > stage II (according to the POP-Q system), post-void residual $>100 \mathrm{ml}$, stress or urge urinary incontinence, diabetes mellitus, a history of pyelonephritis in the past 12 months, interstitial cystitis, symptomatic vulvovaginitis, hormonal therapy, past or current neoplastic disease, urinary tract stones, renal insufficiency.
Women were proposed the use of an oral nutraceutical compound - inulin (2 g), D-mannose (500 mg), cranberry (200 mg), bearberry (200 mg), Olea europaea (100 mg), Orthosiphon (10 mg) and tyndallized Lactobacillus acidophilus SGL 11 (10 billion CFU). The compound was taken per os in the evening before bedtime, not close to meals and with an empty bladder. The therapy was taken for ten days, for 12 months. Patients were invited to withhold urine for a few hours after the intake of the drug and were advised to drink at least 1.5 liters of water per day. Eligible patients were enrolled in two parallel cohorts: subjects using (group 1) or not using (group 2) this nutraceutical compound.

It was recommended to follow a series of hygienicbehavioral rules such as to carry out accurate, intimate hygiene, and to avoid the intake of foods or drinks that irritate the urinary tract (alcohol, coffee, chocolate and spices). Urine culture was performed every month during the follow-up period, prescribing antibiotics to patients with positive results, but without removing them from the study. Follow-up visits were performed by clinicians who were not aware of prescribed treatment. A Visual Analog Scale (VAS) was used to assess the severity of symptoms, where the value 0 indicates the best health state and the value 100 indicates the worst health state. It was performed at the baseline and the 12-month follow-up.

The primary objective of the study was to evaluate the number of women with less than two infective episodes in the 6-month follow-up and less than three episodes in the 12-month follow-up from the beginning of the therapy. The secondary endpoints were to evaluate the reduction of related symptoms and improvement in patients' quality of life at 12-month follow-up compared to baseline.

All charts that were recorded in the database were reviewed carefully by two authors. Data were anonymized before analysis. Institutional Review Board approval was obtained by the local ethic committee. All participants before enrolment signed a comprehensive written consent form. The study conformed to the ethical guidelines of the 1975 Helsinki Declaration. Continuous data were reported as means \pm standard deviation (SD) and analyzed with Student's t-test. Categoric relationships were analyzed by the $\chi^{2}$ test. Probability values of $<0.05$ were considered statistically significant. Statistical analysis was performed with SPSS software (version 19.0; IBM Inc, Armonk, NY). The study was performed according to the Strengthening the Reporting of Observational Studies in Epidemiology (STROBE) guidelines [25].

\section{Results}

One hundred and nineteen women were recruited for the study. The main characteristics of the popula- 
Table 1. Baseline characteristics of the patients

\begin{tabular}{lccc}
\hline Characteristics & $\begin{array}{c}\text { Group 1 } \\
(n=48)\end{array}$ & $\begin{array}{l}\text { Group 2 } \\
(n=46)\end{array}$ & $p$-value \\
\hline Age, years (mean \pm SD) & $53.0 \pm 5.8$ & $54.1 \pm 4.5$ & 0.30 \\
\hline BMI (mean \pm SD) & $24.3 \pm 5.5$ & $25.3 \pm 3.7$ & 0.30 \\
\hline $\begin{array}{l}\text { No. of episodes of cystitis } \\
\text { in the past } 12 \text { months } \\
\text { (mean } \pm S D)\end{array}$ & $3.5 \pm 0.9$ & $3.6 \pm 0.7$ & 0.55 \\
\hline $\begin{array}{l}\text { Patients with UTIs due } \\
\text { to } E \text {. coli }(n / N \text { [\%]) }\end{array}$ & $40 / 48$ & $39 / 46$ & 1.00 \\
\hline VAS score (mean $\pm S D)$ & $68.2 \pm 14.1$ & $70.1 \pm 8.6$ & 0.43 \\
\hline $\begin{array}{l}\text { Daily fluid intake, liter } \\
\text { (mean } \pm S D)\end{array}$ & $1.2 \pm 0.4$ & $1.1 \pm 0.5$ & 0.28 \\
\hline $\begin{array}{l}\text { Data were reported as mean } \pm \text { standard deviation (SD) and analyzed with } \\
\text { Student's } \text {-test. Probability values of }<0.05 \text { were considered statistically } \\
\text { significant }\end{array}$ & &
\end{tabular}

tion are shown in Table 1. No differences were found between the two groups ( $p>0.05$ ).

As seen in Table 1, a large part of patients had their recurrent UTIs caused by E. coli, with similar percentages between the two groups. Other bacteria found were Enterococcus faecalis, Proteus mirabilis, Staphylococcus saprophyticus and Klebsiella pneumoniae. Ninety-four women completed the 12-months follow-up. At 6-month therapy, the reduction in the number of patients with $\geq$ two recurrent UTIs was statistically significant compared to baseline only in group 1 , those receiving combination therapy (Table 2). At 12 months of therapy, the number of patients who were "disease-free" was significantly higher in group 1 compared to those receiving only hydration therapy. No side effects were reported during the follow-up.

The improvement of related symptoms was statistically significant in group 1 at 12-month follow-up, as shown by the reduction in VAS value (Table 3). However, no patients reported adverse events.

\section{Discussion}

The general findings of this study suggested that a combination of D-mannose, inulin, cranberry, bearberry, Olea europaea, Orthosiphon and Lactobacillus acidophilus SGL 11 per os represents a suitable adjuvant option for recurrent UTIs in women wanting a natural approach. UTIs represent a common problem during menopause $[1,26,27]$. About $35-40 \%$ of women experience at least one UTI episode requiring antibiotic treatment, and the most common bacteria is $E$. coli in $85 \%$ of cases [28]. The increase of antibiotic resistance represents an additional emerging issue, particularly for E. coli, Klebsiella, and Enterobacteria strains as they are already resistant to third-generation cephalosporins and carbapenems [2, 29, 30]. Because of the above, researchers are finding suitable adjuvant therapies for UTIs in order to halt this trend. The use of Lactobacillus for UTIs has been widely reported in the literature but the combination with other components such as inulin, D-mannose, cranberry or bearberry represents a new option in this field.

Cianci et al. used Lactobacillus plantarum P 17630 as an adjuvant option to prevent genitourinary infections [31]. The authors found a reduction of recurrent infections in treated women [31]. Another study valued the effect of cranberry, D-mannose, Lactobacillus plantarum and Lactobacillus paracasei in women affected by uncomplicated cystitis. The findings showed a reduction of pain symptoms [32]. However, the use of Lactobacillus crispatus was studied as an intravaginal approach by several authors [33-35].

However, more women claim a natural approach to different diseases, such as menopausal complaints $[9,36,37]$. Notably, in menopause, there is a decrease in estrogens with consequent greater exposure of the mucous membrane of the urinary tract to pathogenic bacteria attacks and therefore the development of cystitis. Drinking at least 1.5 liters of water per day could be a valid therapy, but often it is not enough. It is therefore recommended to follow the standard rules of treatment and prevention: reduce constipation, use antibiotic therapy only if necessary, acidify the urine in order to reduce the bacteria from the bladder mucosa.

However, UTIs present several risk factors such as diabetes, urinary incontinence, disability or recent sexual intercourse [2]. Moreover, this condition severely impacts on daily life in several activities such as social and working, lowering the quality of life [38]. UTIs are also associated with mood changes and anxiety and depression could be experienced by the patients [2, 39, 40]. In older women affected by UTIs, the quality of life could be seriously impaired, especially if urinary incontinence is associated. This aspect is underestimated because patients due to embarrassment did not refer to this condition.

In the last years, the genitourinary syndrome of menopause has claimed more medical attention, especially for this relation to the lowering of quality of life.

Table 2. Patients without recurrent urinary tract infections (UTIS) at 6- and 12-month follow-up compared to baseline

\begin{tabular}{lccc}
\hline Group & \multicolumn{3}{c}{ Patients without recurrent UTIs at 6- and 12-month follow-up compared to baseline } \\
\cline { 2 - 4 } & 6-month follow-up & $p$-value & 12-month follow-up \\
\hline Group $1(n=48)$ & $8 / 48$ & 0.0057 & $11 / 48$ \\
\hline Group $2(n=46)$ & $3 / 46$ & 0.2418 & $4 / 46$ \\
\hline Values are given as $n / N(\%)$. Data were analyzed by the $\chi^{2}$ test. Probability values of $<0.05$ were considered statistically significant
\end{tabular}


This condition is characterized by changes in the urogenital epithelium that reduce the glycogen production and subsequently less Lactobacillus and lactic acid production. In view of the above, an increase in $\mathrm{pH}$ spreads the bacterial colonization.

Our results showed that patients taking the nutraceutical compound, at six months, experienced fewer relapses of UTIs than the control group, and the difference was statistically significant $(<0.05)$. This disparity improved at 12-month follow-up, as shown in Table 2. Our results underline the role of Lactobacillus as well as the other components as effective against bacterial colonization. The restoration of a healthy epithelium decreases the possibility of a relapse. However, the proportion of patients without recurrent UTIs during the follow-up is undoubtedly low and highlights the need for an antibiotic therapy where needed.

Regarding the symptoms experienced by the patients, we noted a statistically significant difference between the two groups $(<0.05)$. In group 1 the VAS score showed a significant decrease at 12-month follow-up, and this finding could be related to cranberry, bearberry and D-mannose. They seem to act as urine acidifiers, and this prevents bacterial adhesion, especially for E. coli. Moreover, pain relief could also be induced by a regulatory effect of Lactobacillus on the urogenital epithelium. D-mannose is a monosaccharide, and it seems able to hinder bacteria adhesion to the urothelium, especially E. coli. E. coli has mannose-sensitive appendices and mannose, binding these in the bladder, favors their detachment from the bladder wall [14-16]. Cranberry can acidify urine and so inhibits the adhesion of microorganisms to the cell membranes of the urinary epithelium. It also inhibits the adhesion of bacteria due to the high content in proanthocyanidins, and favors their elimination through urine [17-19]. Bearberry is a natural antimicrobic for the urinary tract and shows an antimicrobial action on the bladder [20]. Afshar et al., in a randomized clinical trial, observed a reduction of antibiotic use in primary care in women taking bearberry vs the fosfomycin group [41].

The role of constipation is relevant in vaginal infections and has an impact on bacterial colonization. In our study we asked the women to improve several habits such as increasing water daily intake, changing the towel during intimate hygiene and avoiding the intake of foods or drinks that could irritate the urinary tract. The role of inulin and Lactobacillus helped in this way as an anti-constipation effect has been proved [42-44]. Inulin is prebiotic with digestive and intestinal regulating properties $[10,11]$. Lactobacillus acidophilus is a Gram-positive bacterium, able to produce lactic acid as a primary product of glucose fermentation. It has significant beneficial effects for humans, such as the production of compounds inhibiting the growth of other pathogenic microorganisms and the elimination
Table 3. Visual Analog Scale (VAS) score

\begin{tabular}{lccc}
\hline Group & \multicolumn{3}{c}{ VAS score } \\
\cline { 2 - 4 } & Baseline & 12-month follow-up & $p$-value \\
\hline Group 1 $(n=48)$ & $68.2 \pm 14.1$ & $45.2 \pm 7.6$ & 0.0001 \\
\hline Group 2 $(n=46)$ & $70.1 \pm 8.6$ & $66.4 \pm 9.5$ & 0.0533 \\
\hline
\end{tabular}

Comparison between group 1 and group 2 at baseline and at 12-month follow-up. Data were reported as mean \pm standard deviation (SD) and analyzed with Student's $t$-test. Probability values of $<0.05$ were considered statistically significant

of toxins produced by proteolytic bacteria [12, 13]. An anti-constipation effect has also been demonstrated for Olea europaea, the common olive tree. It is rich in oleuropein, a bitter glucoside especially present in the leaves. For this reason, olive leaves have depurative, anti-inflammatory and laxative properties [21, 22]. Orthosiphon, also known as Java tea, is helpful for UTIs thanks to polymethylated flavones of lipophilic nature that reduce bacterial colonization [23, 24].

The strength of our study is the 12-month follow-up period and presence of two study groups similar in age, $\mathrm{BMI}$, symptoms, and daily fluid intake at the recruitment. A point to underline is that the use of a nutraceutical approach is useful in non-complicated cases such as not highly symptomatic patients or a recent history of UTIs. In other cases, an antibiotic approach should be considered.

However, we have to underline the limitations of the study. It was an observational study, and such potential biases should be considered. Moreover, the study population was small and further studies, especially randomized controlled trials, are needed to clarify the efficacy of this nutraceutical compound on UTIs.

\section{Conclusions}

Based on the above evidence, oral therapy with D-mannose, inulin, cranberry, bearberry, Olea europaea, Orthosiphon and Lactobacillus acidophilus SGL 11 appears to be a useful and risk-free option for recurrent UTIs in women wanting a natural approach. However, further studies should compare its effectiveness with that of common antibiotics prescribed to treat UTIs in menopausal women to investigate its efficacy as an alternative to antibiotic therapy.

\section{Disclosure}

The authors report no conflict of interest.

\section{References}

1. Gupta K, Grigoryan L, Trautner B. Urinary Tract Infection. Ann Intern Med 2017; 167: ITC49-ITC64.

2. Mody L, Juthani-Mehta M. Urinary tract infections in older women: a clinical review. JAMA 2014; 311: 844-854. 
3. Faubion SS, Sood R, Kapoor E. Genitourinary Syndrome of Menopause: Management Strategies for the Clinician. Mayo Clin Proc 2017; 92: 1842-1849.

4. Szadowska-Szlachetka ZC, Stasiak E, Leziak A, et al. Intensity of menopausal symptoms and quality of life in climacteric women. Prz Menopauzalny 2019; 18: 217-221.

5. Portman DJ, Gass MLS. Vulvovaginal Atrophy Terminology Consensus Conference Panel Genitourinary syndrome of menopause: new terminology for vulvovaginal atrophy from the International Society for the Study of Women's Sexual Health and the North American Menopause Society. Menopause 2014; 21: 1063-1068.

6. Augoulea A, Moros M, Kokras N, et al. Association of menopausal symp toms with sociodemographic factors and personality traits. Prz Menopauzalny 2019; 18: 191-197.

7. Anger J, Lee U, Ackerman AL, et al. Recurrent Uncomplicated Urinary Tract Infections in Women: AUA/CUA/SUFU Guideline. J Urol 2019; 202 282-289.

8. Guglietta A. Recurrent urinary tract infections in women: risk factors, etiology, pathogenesis and prophylaxis. Future Microbiol 2017; 12: 239246.

9. Torella M, Del Deo F, Grimaldi A, et al. Efficacy of an orally administered combination of hyaluronic acid, chondroitin sulfate, curcumin and quercetin for the prevention of recurrent urinary tract infections in postmenopausal women. Eur J Obstet Gynecol Reprod Biol 2016; 207: 125-128.

10. Bustamante M, Oomah BD, Oliveira WP, et al. Probiotics and prebiotics potential for the care of skin, female urogenital tract, and respiratory tract. Folia Microbiol (Praha) 2020; 65: 245-264.

11. Aragón IM, Herrera-Imbroda B, Queipo-Ortuño MI, et al. The Urinary Tract Microbiome in Health and Disease. Eur Urol Focus 2018; 4: 128138.

12. Shim YH, Lee SJ, Lee JW. Antimicrobial activity of lactobacillus strains against uropathogens. Pediatr Int 2016; 58: 1009-1013.

13. Homayouni A, Bastani P, Ziyadi S, et al. Effects of probiotics on the recurrence of bacterial vaginosis: a review. J Low Genit Tract Dis 2014, 18: 79-86

14. Kranjčec B, Papeš D, Altarac S. D-mannose powder for prophylaxis of recurrent urinary tract infections in women: a randomized clinical trial. World J Urol 2014; 32: 79-84.

15. Domenici L, Monti M, Bracchi C, et al. D-mannose: a promising support for acute urinary tract infections in women. A pilot study. Eur Rev Med Pharmacol Sci 2016; 20: 2920-2925.

16. Sihra N, Goodman A, Zakri R, et al. Nonantibiotic prevention and management of recurrent urinary tract infection. Nat Rev Urol 2018; 15: 750-776.

17. Fu Z, Liska D, Talan D, Chung M. Cranberry Reduces the Risk of Urinary Tract Infection Recurrence in Otherwise Healthy Women: A Systematic Review and Meta-Analysis. J Nutr 2017; 147: 2282-2288.

18. Nabavi SF, Sureda A, Daglia M, et al. Cranberry for Urinary Tract Infec tion: From Bench to Bedside. Curr Top Med Chem 2017; 17: 331-339.

19. Rauf A, Imran M, Abu-Izneid T, et al. Proanthocyanidins: A comprehensive review. Biomed Pharmacother 2019; 116: 108999.

20. Moore M, Trill J, Simpson C, et al. Uva-ursi extract and ibuprofen as alternative treatments for uncomplicated urinary tract infection in women (ATAFUTI): a factorial randomized trial. Clin Microbiol Infect 2019; 25: $973-980$

21. Gorzynik-Debicka M, Przychodzen P, Cappello F, et al. Potential Health Benefits of Olive Oil and Plant Polyphenols. Int J Mol Sci 2018; 19: 686.

22. Yuan J-J, Wang C-Z, Ye J-Z, et al. Enzymatic hydrolysis of oleuropein from Olea europea (olive) leaf extract and antioxidant activities. Molecules 2015; 20: 2903-2921.

23. Ashraf K, Sultan S, Adam A. Orthosiphon stamineus Benth. is an Outstanding Food Medicine: Review of Phytochemical and Pharmacological Activities. J Pharm Bioallied Sci 2018 10: 109-118.

24. Bokhari RA, Lau SF, Mohamed S. Orthosiphon stamineus (Misai Kucing) ameliorated postmenopausal osteoporosis in rat model. Menopause 2018; 25: 202-210.

25. von Elm E, Altman DG, Egger M, et al. STROBE Statement: linee guida per descrivere gli studi osservazionali. Ter Evid Based 2008; 1: 1-8.

26. Salvatore S, Salvatore $S$, Cattoni E, et al. Urinary tract infections in women. Eur J Obstet Gynecol Reprod Biol 2011; 156: 131-136.
27. Torella M, Schettino MT, De Franciscis P, et al. Intravesical therapy in recurrent cystitis: a multi-center experience. J Infect Chemother 2013 19: 920-925.

28. Foxman B, Gillespie B, Koopman J, et al. Risk Factors for Second Urinary Tract Infection among College Women. Am J Epidemiol 2000; 151: 1194-1205.

29. Selekman RE, Shapiro DJ, Boscardin J, et al. Uropathogen Resistance and Antibiotic Prophylaxis: A Meta-analysis. Pediatrics 2018; 142 e20180119.

30. Tamadonfar KO, Omattage NS, Spaulding CN, Hultgren SJ. Reaching the End of the Line: Urinary Tract Infections. Microbiol Spectr 2019; 7: BAI-0014-2019.

31. Cianci A, Cicinelli E, De Leo V, et al. Observational prospective study on Lactobacillus plantarum P 17630 in the prevention of vaginal infections, during and after systemic antibiotic therapy or in women with recurrent vaginal or genitourinary infections. J Obstet Gynaecol 2018, 38: 693-696.

32. Vicariotto F. Effectiveness of an association of a cranberry dry extract, D-mannose, and the two microorganisms Lactobacillus plantarum LP01 and Lactobacillus paracasei LPC09 in women affected by cystitis: a pilot study. J Clin Gastroenterol 2014; 48: S96-101.

33. Ngugi BM, Hemmerling A, Bukusi EA, et al. Effects of bacterial vaginosis-associated bacteria and sexual intercourse on vaginal colonization with the probiotic Lactobacillus crispatus CTV-05. Sex Transm Dis 2011, 38: 1020-1027.

34. Stapleton AE, Au-Yeung M, Hooton TM, et al. Randomized, PlaceboControlled Phase 2 Trial of a Lactobacillus crispatus Probiotic Given Intravaginally for Prevention of Recurrent Urinary Tract Infection. Clin Infect Dis 2011; 52: 1212-1217.

35. Bohbot JM, Daraï E, Bretelle F, et al. Efficacy and safety of vaginally administered lyophilized Lactobacillus crispatus IP 174178 in the prevention of bacterial vaginosis recurrence. J Gynecol Obstet Hum Reprod 2018; 47: 81-86

36. De Franciscis P, Colacurci N, Riemma G, et al. A Nutraceutical Approach to Menopausal Complaints. Medicina 2019; 55: 544.

37. Rizzo G, Garzon S, Fichera M, et al. Vitamin D and Gestational Diabetes Mellitus: Is There a Link? Antioxidants (Basel) 2019; 8: 511.

38. Medina M, Castillo-Pino E. An introduction to the epidemiology and burden of urinary tract infections. Ther Adv Urol 2019; 11: 1756287219832172

39. Renard J, Ballarini S, Mascarenhas T, et al. Recurrent Lower Urinary Tract Infections Have a Detrimental Effect on Patient Quality of Life: a Prospective, Observational Study. Infect Dis Ther 2015; 4: 125-135.

40. Riemma G, Schiattarella A, La Verde M, et al. Efficacy of Low-Dose Paroxetine for the Treatment of Hot Flushes in Surgical and Physiological Postmenopausal Women: Systematic Review and Meta-Analysis of Randomized Trials. Medicina (Kaunas) 2019; 55: 554.

41. Afshar K, Fleischmann N, Schmiemann G, et al. Reducing antibiotic use for uncomplicated urinary tract infection in general practice by treatment with uva-ursi (REGATTA) - a double-blind, randomized, controlled comparative effectiveness trial. BMC Complement Altern Med 2018; 18: 203.

42. Collado Yurrita L, San Mauro Martín I, Ciudad-Cabañas MJ, et al. Effectiveness of inulin intake on indicators of chronic constipation; a metaanalysis of controlled randomized clinical trials. Nutr Hosp 2014; 30 : 244-252.

43. Kaur N, Gupta AK. Applications of inulin and oligofructose in health and nutrition. J Biosci 2002; 27: 703-714.

44. Vitale SG, Caruso S, Rapisarda AMC, et al. Isoflavones, calcium, vitamin $D$ and inulin improve quality of life, sexual function, body composition and metabolic parameters in menopausal women: result from a prospective, randomized, placebo-controlled, parallel-group study. Prz Menopauzalny 2018; 17: 32-38. 